\section{Domiciliary long-term non- invasive ventilation in COPD: should we select subgroups with a better likelihood to respond to NIV in subsequent randomised controlled trials?}

The efficacy of non-invasive ventilation (NIV) for severe COPD exacerbation has been unambiguously demonstrated. The study by Struik et al recently published in Thorax ${ }^{1}$ demonstrates that pursuing nocturnal NIV after acute respiratory failure in patients with COPD exhibiting prolonged hypercapnia $>48 \mathrm{~h}$ after termination of ventilatory support does not reduce time to readmission or rate of deaths at 1 year. They included a mixed cohort of patients with acute-on-chronic and acute respiratory failures. However, in future studies it may be interesting to select patients with persistent hypercapnia several weeks after the acute exacerbation episode. Nevertheless, long-term NIV efficacy for stable COPD remains uncertain in terms of hard outcomes. Clinical cohort studies suggest that mortality and readmission rates under NIV are dependent upon the underlying COPD subgroup. ${ }^{23}$

We recently showed that a reduction in risk of death and hospital readmission is obtained only in obese patients with COPD with NIV usage $>5 \mathrm{~h} /$ day. $^{2}$ In the Struik study, ${ }^{1}$ randomisation was stratified on body mass index although the mean body mass index of the whole cohort was $25 \mathrm{kgm}^{-2}$ (SD around $6 \mathrm{kgm}^{-2}$ ). The majority of Struik's cohort had 'respiratory COPD' characterised by severe airflow limitation without obesity, ${ }^{4}$ a subgroup supposed to be less responsive to $\mathrm{NIV}^{2}$

Obesity is a main risk factor of sleep apnoea, and patients with overlap syndrome (COPD+sleep apnoea) benefit from positive pressure treatment. ${ }^{5}$ We obtained positive results with NIV in obese patients with COPD after adjustment for all independent risk factors associated with prognosis, particularly sleep apnoea. This suggests that NIV, beyond correcting sleep apnoea, may also lessen the deleterious impact of nocturnal hypoventilation and improve respiratory mechanics.

In Struik's study, ${ }^{1}$ in both intention to treat and per protocol analyses (threshold for NIV compliance $\geq 5 \mathrm{~h} /$ night) NIV was not effective on time to readmission or death. In our work, we analysed the impact of daily use of NIV as a time-dependent variable without any pre-existing arbitrary threshold. We found a U-shaped curve between NIV usage and risk of readmission or death. This suggests that NIV adherence should be assessed as a continuous variable rather than with a predefined threshold.

In summary, we suggest that subsequent randomised controlled trials be conducted on specific COPD subgroups that have better likelihood of responding to NIV (ie, obese patients with comorbidities and moderate to severe airflow obstruction). This might represent a new approach to the challenging area of long-term NIV in stable patients with COPD. Jean-Christian Borel, ${ }^{1,2}$ Christophe Pison, ${ }^{3,4,5}$
Jean-François Timsit, ${ }^{6,7}$ Jean-Louis Pepin

${ }^{1}$ Univ Grenoble Alpes, HP2 INSERM U1042, Grenoble, France

${ }^{2} \mathrm{AGIR}$ à dom, Non Profit Home Care Provider, Meylan, France

${ }^{3}$ Clinique Universitaire de Pneumologie, Pôle Thorax et Vaisseaux, Grenoble University Hospital, Grenoble, France

${ }^{4}$ European Institute for Systems Biology and Medicine, EISBM, Lyon, France

${ }^{5}$ INSERM 1055, Univ Grenoble Alpes, Grenoble, France

${ }^{6}$ Service de Réanimation Médicale, Pôle Urgences

Médecine Aigüe, Grenoble University Hospital,

Grenoble, France

${ }^{7}$ Service de Réanimation Médicale, Hôpital BichatAPHP, Paris, France

${ }^{8}$ Clinique Universitaire de Physiologie et Sommeil, Pôle Thorax et Vaisseaux, Grenoble University Hospital, Grenoble, France

Correspondence to Dr Jean-Christian Borel, AGIR à dom Association, 19, bd de la Chantourne, $38700 \mathrm{La}$ Tronche-France; j.bore@@agiradom.com

Acknowledgement The authors thank Dr Alison Foote for English editing.

Contributors J-LP received personal fees from RESMED and grants from RESMED, PHILIPS and AGIRadom. These fees and grants are outside the submitted work. CP has received grants from AGIRadom, a non-profit home care company. These grants are outside the submitted work.

Competing interests $\mathrm{J}-\mathrm{CB}$ is employed by AGIRadom, a non-profit home care company.

Provenance and peer review Not commissioned; externally peer reviewed.

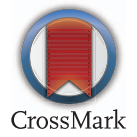

To cite Borel J-C, Pison C, Timsit J-F, et al. Thorax 2014;69:1143.

Received 25 June 2014

Accepted 17 July 2014

Published Online First 1 August 2014

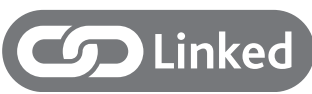

http://dx.doi.org/10.1136/thoraxjnl-2014-205126

http://dx.doi.org/10.1136/thoraxjnl-2014-206066

Thorax 2014;69:1143.

doi:10.1136/thoraxjnl-2014-205949

\section{REFERENCES}

1 Struik FM, Sprooten RT, Kerstjens HA, et al. Nocturnal non-invasive ventilation in COPD patients with prolonged hypercapnia after ventilatory support for acute respiratory failure: a randomised, controlled, parallel-group study. Thorax 2014;69:826-34.

2 Borel JC, Pepin JL, Pison C, et al. Long term adherence with non-invasive ventilation improves prognosis in obese COPD patients. Respirology 2014;19:857-65.

3 Carrillo A, Ferrer M, Gonzalez-Diaz G, et al. Noninvasive ventilation in acute hypercapnic respiratory failure caused by obesity hypoventilation syndrome and chronic obstructive pulmonary disease. Am J Respir Crit Care Med 2012;186:1279-85.

4 Garcia-Aymerich J, Gomez FP, Benet M, et al. Identification and prospective validation of clinically relevant chronic obstructive pulmonary disease (COPD) subtypes. Thorax 2011;66:430-7.

5 Marin JM, Soriano JB, Carrizo SJ, et al. Outcomes in patients with chronic obstructive pulmonary disease and obstructive sleep apnea: the overlap syndrome. Am J Respir Crit Care Med 2010;182:325-31. 